\title{
Importância do pré-natal odontológico: uma revisão narrativa
}

\author{
Importance of dental prenatal care: a narrative review \\ Importancia de la atención dental prenatal: una revisión narrativa
}

Recebido: 04/11/2021 | Revisado: 11/11/2021 | Aceito: 13/11/2021 | Publicado: 22/11/2021

\author{
Érika Dayanne Alves da Silva \\ ORCID: https://orcid.org/0000-0001-6055-9787 \\ Faculdade Integrada Carajás, Brasil \\ E-mail: erikadayanne.love@hotmail.com \\ Malba Rayanne Pereira da Silva \\ ORCID: https://orcid.org/0000-0001-9411-0400 \\ Faculdade Integrada Carajás, Brasil \\ E-mail: malbarayanne@ hotmail.com \\ Yolanda de Jesus Morais \\ ORCID: https://orcid.org/0000-0001-7105-8267 \\ Universidade Federal do Pará, Brasil \\ E-mail: yolandamorais123@gmail.com \\ Poliana Albino Kervahal \\ ORCID: https://orcid.org/0000-0002-8749-9638 \\ Centro Universitário Luterano de Palmas, Brasil \\ E-mail: polianakervahalodonto@gmail.com
}

\begin{abstract}
Resumo
Introdução: No pré-natal odontológico, o cirurgião dentista irá acompanhar a gestante, reconhecendo suas necessidades odontológicas e deverá orientar e tratar a mãe sobre a higiene oral para prevenir complicações, como cáries, gengivite, periodontite, ou até mesmo o parto prematuro. Esse estudo tem como objetivo apresentar a necessidade do atendimento odontológico no pré-natal, descrevendo a importância dos cuidados odontológicos na gestação. Metodologia: Foram utilizados os seguintes descritores e suas combinações: "cuidado pré-natal" e "odontologia" nas bases de dados SCIELO, LILACS e Google Acadêmico. Foram selecionados, inicialmente, um total de 40 artigos relacionados ao tema, dentre esses artigos, 22 foram selecionados após leitura completa. Resultados e Discussões: O pré-natal odontológico precisa ser expandido, mas para que o mesmo cresça e ajude as futuras mães sobre a importância do mesmo e preciso os profissionais adquiriram conhecimento teórico, prático e cientifico sobre o assunto. Considerações finais: Observou-se que os estudos se posicionaram positivamente sobre a importância do acesso aos serviços odontológicos, orientações e aconselhamento precoce sobre a saúde bucal da gestante, enfatizando de que forma a mesma pode influenciar diretamente na saúde geral do bebê.
\end{abstract}

Palavras-chave: Cuidado pré-natal; Odontologia; Gestantes.

\begin{abstract}
Introduction: In dental prenatal care, the dental surgeon will accompany the pregnant woman, recognizing her dental needs and should guide and treat the mother about oral hygiene to prevent complications such as caries, gingivitis, periodontitis, or even premature birth. This study aims to present the need for prenatal dental care, describing the importance of dental care during pregnancy. Methodology: The following descriptors and their combinations were used: "prenatal care" and "dentistry" in the SCIELO, LILACS and Academic Google databases. Initially, a total of 40 articles related to the topic were selected, among these articles, 22 were selected after full reading. Results and Discussions: Dental prenatal care needs to be expanded, but for it to grow and help future mothers about its importance and accuracy, professionals have acquired theoretical, practical and scientific knowledge on the subject. Final considerations: It was observed that the studies were positively positioned on the importance of access to dental services, guidance and early counseling on the oral health of pregnant women, emphasizing how it can directly influence the general health of the baby.
\end{abstract}

Keywords: Prenatal care; Dentistry; Pregnant women.

\section{Resumen}

Introducción: En la atención prenatal dental, el cirujano dentista acompañará a la gestante, reconociendo sus necesidades dentales y debe orientar y tratar a la madre sobre la higiene bucal para prevenir complicaciones como caries, gingivitis, periodontitis o incluso parto prematuro. Este estudio tiene como objetivo presentar la necesidad de 
atención odontológica prenatal, describiendo la importancia de la atención odontológica durante el embarazo. Metodología: Se utilizaron los siguientes descriptores y sus combinaciones: "atención prenatal” y "odontología" en las bases de datos SCIELO, LILACS y Academic Google. Inicialmente se seleccionaron un total de 40 artículos relacionados con el tema, entre estos artículos se seleccionaron 22 luego de la lectura completa. Resultados y Discusiones: Es necesario ampliar la atención odontológica prenatal, pero para que ésta crezca y ayude a las futuras madres sobre su importancia y precisión, los profesionales han adquirido conocimientos teóricos, prácticos y científicos sobre el tema. Consideraciones finales: Se observó que los estudios se posicionaron positivamente sobre la importancia del acceso a los servicios dentales, la orientación y el asesoramiento temprano sobre la salud bucal de la gestante, enfatizando cómo puede influir directamente en la salud general del bebé.

Palabras clave: Atención prenatal; Odontología; Mujeres embarazadas.

\section{Introdução}

A gestação é um período singular na vida da mulher, pois ela está vivendo um momento de muitas expectativas em torno da nova vida que está se formando dentro dela. A gravidez também traz várias mudanças fisiológicas, psicológicas, emocionais e sociais, sendo, portanto, um período de transformações que possibilita o surgimento de patologias orais que podem ser capazes de trazer malefícios para a saúde da mãe e do bebê (Alves \& Bezerra, 2020).

No pré-natal odontológico, o cirurgião dentista irá acompanhar a gestante, reconhecendo suas necessidades odontológicas e deverá orientar e tratar a mãe sobre a higiene oral para prevenir complicações, como cáries, gengivite, periodontite, ou até mesmo o parto prematuro (Botelho, Lima, Barros \& Almeida 2019).

A equipe multidisciplinar que recebe as mulheres grávidas no primeiro momento tem o dever de reconhecer e orientar as gestantes. Assim, deparamo-nos com uma pergunta: qual é a importância do cirurgião dentista no pré-natal?

Portanto, entende-se que este estudo possa ter um impacto positivo na assistência ao pré-natal, proporcionando um maior aprimoramento teórico sobre as necessidades da gestante e a importância do cirurgião dentista nessa fase, na qual as maiorias das enfermidades bucais podem surgir. Trata-se, então, de um período propício para motivar comportamentos preventivos e educativos que possibilitem à paciente mudanças de hábitos em saúde.

Esse estudo tem como objetivo apresentar a necessidade do atendimento odontológico no pré-natal, descrevendo a importância dos cuidados odontológicos na gestação. Além disso, deve-se pontuar os problemas odontológicos que possam surgir na gestação e conscientizar os profissionais cirurgiões dentistas sobre as necessidades de um atendimento humanizado e de qualidade para esse público-alvo.

\section{Pré-natal Odontológico}

O objetivo do acompanhamento pré-natal é assegurar o desenvolvimento da gestação, permitindo o parto de um recém-nascido saudável, sem impacto para a saúde materna, inclusive com a abordagem dos aspectos psicossociais e das atividades educativas e preventivas. Dessa forma, propicia-se à gestante segurança e bem-estar, favorecendo certo equilíbrio emocional quanto aos cuidados odontológicos e sua necessidade durante esse período em que a mulher grávida se encontra vulnerável às diversas manifestações bucais (Lopes et al., 2018; Reis et al., 2010).

A maioria dos procedimentos odontológicos pode ser realizada durante a gravidez, observando-se alguns cuidados: planejar sessões curtas, adequar à posição da cadeira e evitar consultas matinais, já que neste período as gestantes têm mais ânsia de vômito e risco de hipoglicemia. Exodontias não complicadas, tratamentos periodontal e endodôntico, restaurações dentárias, instalação de próteses e outros tipos de procedimentos devem ser realizados com segurança, de preferência no segundo trimestre. Tratamentos seletivos, como as reabilitações bucais extensas e as cirurgias mais invasivas podem ser programados para o período de pós-parto (Bastiani, Cota, Provenzano, Fracasso, Honório \& Dias, 2013). 
Outros fatores também parecem estar associados a um menor acesso e utilização dos serviços odontológicos por gestantes, entre eles o nível socioeconômico, desinteresse, falta de tempo e necessidade de repousar devido à hipertensão, impossibilitando o deslocamento para a consulta. Quanto à escolaridade das mães, observa-se que independentemente do nível, a maioria recusa-se ao tratamento odontológico no período gestacional, evidenciando que a falta de informação sobre o tema não é restrita às gestantes com menor nível educacional (Silva \& Savian, 2020).

É importante ouvir as gestantes nos grupos operativos para saber quais os seus anseios, conhecer seus problemas, suas crenças, respeitando-as e respondendo-as com linguagens claras e objetivas, considerando sua opinião e tendo o cuidado para não se demonstrar autoritário. Deve-se mostrar as mudanças que ocorrem na boca durante a gravidez, enfatizando a necessidade de higiene, estimulando o autocuidado e hábitos de vida saudável. Também é possível fornecer outras informações, como: uso de medicamentos sem prescrição de um especialista, fumos, drogas e seus efeitos sobre a saúde bucal e gestacional (Brasil, 2008).

Portanto, a saúde bucal da gestante ainda é cercada de muitas dúvidas e crenças passadas ao longo dos tempos por meio de experiências relatadas por familiares e outras gestantes, já que estas desconhecem fatores biomédicos importantes referentes à saúde bucal, não colocando o pré-natal odontológico como prioridade, o que contribui para o agravo de problemas bucais e para a precariedade das condições bucais tanto das mães quanto de seus filhos (Botelho Botelho, Lima, Barros \& Almeida, 2019).

\section{Problemas odontológicos na gestação}

Como se sabe, os hormônios estrógeno e progesterona se elevam para manter a gestação, logo, a resposta inflamatória exacerbada se dá pela atividade dos agentes irritantes locais, como a placa bacteriana (Harb, Carmo \& Boaventura, 2020). A cárie dentária e as doenças gengivais e periodontais têm alta prevalência entre gestantes, apresentando mecanismos biológicos que podem afetar a gestação, sendo considerados fatores de risco para a pré-eclâmpsia e para o nascimento de bebês prematuros e com baixo peso (Silva \& Vieira, 2020; Moimaz, Saliba, Santos, Queiroz \& Garbin, 2011). Além disso, a doença periodontal (DP) afeta entre 15-30\% da população em idade fértil, com uma proporção relativamente elevada em mulheres grávidas (Ebrahim, Oliveira, Peres \& Franco, 2014).

O diabetes mellitus compreende um grupo de doenças metabólicas, resultante de um defeito na secreção e/ou na ação da insulina, caracterizado pela hiperglicemia ou pelo aumento dos níveis de glicose no sangue. Pode ser classificado, de acordo com a etiologia, em: tipo 1, tipo 2, gestacional e outros tipos específicos. O diabetes gestacional é definido como qualquer grau de intolerância à glicose com início ou primeiro reconhecimento durante a gravidez (Sousa, Nóbrega \& Araki, 2014).

Diversas teorias tentam correlacionar a saúde periodontal de gestantes com RN prematuros e/ou com baixo peso. Uma das hipóteses mais aceitas é de que a infecção e a inflamação periodontal são veículos para organismos Gram-negativos e lipopolissacarídeos, que podem se disseminar no organismo por meio de grandes vasos, elevando os mediadores inflamatórios, como PGE2 (Prostaglandina E2) e TNF (Fator de Necrose Tumoral), tornando-se, assim, uma ameaça potencial à placenta fetal (Mascarenhas, Vilarinho, Moura, Moura \& Ferro, 2012).

O tumor gravídico, ou granuloma piogênico, é uma lesão benigna que surge geralmente no priᄀmeiro trimestre da gestação e acomete mais de 5\% das gestantes. Pode resultar de traumatismos repetitivos e irritação local sobre os tecidos gengivais e ocorre preferencialmente na região anterior da maxila (Ebrahim, Oliveira, Peres \& Franco, 2014).

O granuloma piogênico é uma das entidades mais frequentemente associadas à expansão dos tecidos moles, representando entre $3,8 \%$ e $7 \%$ de todas as biópsias de lesões realizadas na cavidade bucal. Semelhante a um tumor, de 
natureza não neoplásica, considera-se que represente uma resposta tecidual exuberante a uma irritação local ou trauma. No entanto, a patogênese do granuloma piogênico ainda não se apresenta completamente estabelecida (Kruger, Rosa, Pappen, Romano \& Corrêa, 2014).

Há, ainda, uma predileção pelo sexo feminino, sendo as pacientes com cor da pele branca as mais afetadas e o sítio mais comum à gengiva. As lesões, em sua maioria, são descritas como nódulos com base pediculada, de consistência macia e superfície avermelhada. O tamanho pode variar de 1 a 6 cm, com uma média de 1,3 cm (Kruger, Rosa, Pappen, Romano \& Corrêa, 2014).

Alterações no fluxo salivar e composição da saliva também podem ocorrer durante a gravidez. Essas alterações podem estar relacionadas à constantes variações hormonais, no entanto, existem controversas sobre o assunto. A partir de um levantamento bibliográfico, observaram-se as discordâncias em relação aos parâmetros salivares de gestantes, como a taxa de fluxo salivar, pH e capacidade tampão da saliva e as sensações de xerostomia e paregeusia. Assim, justificou-se investigar esses parâmetros salivares com atenção para o comprometimento da saúde bucal diante de alterações salivares (Leal, Rolim, Muniz \& Farias, 2013).

salivares com atenção para o comprometimento da saúde bucal diante de alterações salivares (Leal, Rolim, Muniz \& Farias, 2013).

\section{Protocolo de atendimento odontológico}

O protocolo de atendimento é constituído de anamnese, exame clínico, preenchimento do odontograma, orientações sobre higiene bucal, dieta, mitos e verdades sobre o atendimento odontológico, amamentação, hábitos de sucção não nutritivos, cuidados com o bebê, realização de escovação supervisionada e agendamento de retorno para realização do tratamento (Silva, 2013).

\section{Exame Radiográfico}

O exame radiográfico não precisa ser evitado durante a gestação, uma vez que a quantidade de radiação que a mãe é exposta para a tomada radiográfica periapical é muito menor que a dose necessária para ocasionar uma malformação congênita, visto que o feto recebe $1 / 50000$ da exposição direta da cabeça da mãe (Silva, 2013).

\section{Medicações durante a gestação}

A automedicação é uma prática difundida em diversos países, inclusive no Brasil e se constitui com um dos principais problemas na gravidez, principalmente pelo desconhecimento das gestantes quanto aos riscos que esta prática acarreta (Silva, 2013).

A mepivacaína deve ser evitada durante a gestação e lactação devido à imaturidade do sistema enzimático hepático do feto e do bebê, o que dificulta a metabolização correta deste fármaco, além de ser rapidamente absorvida e normalmente ser provida de uma solução mais concentrada quando indicada sem vasoconstritor. Os Quadros 1 e 2 classificam os anestésicos locais dentro das cinco categorias de risco segundo a agência norte-americana Food and Drug Administration FDA, assim como a segurança no uso dos mesmos durante a gravidez e a amamentação (Rodrigues, Mármora, Carrion, Rego \& Pospich, 2017) 
Quadro 1 - Classificação dos medicamentos em categorias de risco segundo a FDA.

\begin{tabular}{|l|l|}
\hline Medicamentos pertencentes à CATEGORIA A & $\begin{array}{l}\text { Estudos controlados em humanos não indicam riscos aparentes para o } \\
\text { feto. }\end{array}$ \\
\hline Medicamentos pertencentes à CATEGORIA B & $\begin{array}{l}\text { Estudos em animais controlados não indicam riscos para o feto, mas ainda } \\
\text { sem estudos confiáveis em mulheres grávidas. }\end{array}$ \\
\hline Medicamentos pertencentes à CATEGORIA C & $\begin{array}{l}\text { Estudos em animais mostraram efeitos adversos para o feto, mas não existem } \\
\text { estudos em humanos. }\end{array}$ \\
\hline Medicamentos pertencentes à CATEGORIA D & $\begin{array}{l}\text { Evidências positivas de risco fetal humano, mas cujos benefícios podem } \\
\text { justificar o uso. }\end{array}$ \\
\hline Medicamentos pertencentes à CATEGORIA X & $\begin{array}{l}\text { Evidências positivas de anormalidades fetais, com contraindicações tanto em } \\
\text { mulheres grávidas quanto nas que querem engravidar, pois os riscos superam } \\
\text { os benefícios. }\end{array}$ \\
\hline
\end{tabular}

Fonte: https://periodicos.unemat.br/index.php/jhnpeps/article/view/1835/1675

Quadro 2 - Classificação dos agentes anestésicos quanto à segurança.

\begin{tabular}{|l|c|l|l|}
\hline \multicolumn{1}{|c|}{ Agente } & $\begin{array}{c}\text { Classificação } \\
\text { segundo a FDA }\end{array}$ & \multicolumn{1}{c|}{$\begin{array}{c}\text { Segurança no uso durante a } \\
\text { gravidez }\end{array}$} & \multicolumn{1}{c|}{$\begin{array}{c}\text { Segurança no uso durante a } \\
\text { amamentação }\end{array}$} \\
\hline Articaína & C & Uso com cuidado & Uso com cuidado \\
\hline Bupivacaína & C & Uso com cuidado & Sim \\
\hline Lidocaína & B & Sim & Sim \\
\hline Mepivacaína & C & Uso com cuidado & Sim \\
\hline Prilocaína & B & Uso com cuidado & Uso com cuidado \\
\hline Benzocaína (tópica) & C & Uso com cuidado & Sim \\
\hline Lidocaína (tópica) & B & Sim & Uso com cuidado \\
\hline Tetracaína (tópica) & C & Uso com cuidado & \\
\hline
\end{tabular}

Fonte: https://periodicos.unemat.br/index.php/jhnpeps/article/view/1835/1675.

\section{Conscientização dos profissionais odontólogos}

Devido a essas questões e peculiaridades, surge uma série de dúvidas no profissional e na paciente no que se refere aos tipos de procedimentos odontológicos que podem ser executados durante a gestação (Martins, Pinheiro, Arantes, Nascimento \& Júnior, 2013).

Ressalta-se que o primeiro trimestre se mostra o período menos adequado para o tratamento odontológico, pois nele ocorrem as principais transformações embriológicas. O segundo trimestre é o período mais adequado para intervenções clínicas e procedimentos odontológicos essenciais, sempre de acordo com as indicações. Durante esse período, a organogênese está completa e o feto já está desenvolvido (Botelho, Lima, Barros \& Almeida, 2019).

A gestante pode ser atendida em qualquer período gestacional, embora o segundo trimestre seja o mais seguro. Outra dúvida frequente é em relação ao protocolo de uso de anestésicos. A maioria dos profissionais utilizam a lidocaína sem vasoconstritor como anestésico de escolha para gestantes. Porém, a literatura aponta a lidocaína como anestésico de uso seguro durante a gestação, sendo a lidocaína a $2 \%$ associada ao vasoconstritor adrenalina considerada a mais segura solução 
anestésica para pacientes gestantes saudáveis, considerando a dosagem adequada (Carvalho, Fritzen, Parodes, Santos \& Gedoz, 2013).

O vasoconstritor é absorvido lentamente, aumentando a durabilidade do efeito anestésico, o que proporciona mais conforto para as pacientes. Por isso, é tão importante que o profissional tenha conhecimento sobre os protocolos de intervenções durante o pré-natal e seja criterioso na realização da anamnese e avalie a história médica de gestações anteriores, intercorrências gestacionais, estado psicológico e aceitação da gravidez, para melhor atender as gestantes (Martins, Pinheiro, Arantes, Nascimento \& Júnior, 2013; Vasconcelos, Vasconcelos, Mafra, Júnior, Queiroz \& Barboza, 2012).

Ainda, corroborando com este fato, há profissionais que consideram adiar ou mesmo não realizar procedimentos clínicos no período gestacional, por desconhecimento ou receio de causar algum agravo à saúde do binômio mãe-bebê (Silva \& Vieira, 2020).

\section{Metodologia}

Este artigo é uma revisão narrativa da literatura, de caráter qualitativo, explicativo e dedutivo (Estrela, 2018), no qual aborda a importância do pré-natal odontológico.

Para a seleção dos artigos nesta revisão, foram estabelecidos os seguintes critérios de inclusão: publicações entre 2010 a 2021, na língua portuguesa e inglesa, que tivessem como tema a importância do atendimento odontológico no pré-natal.

Como critérios de exclusão, foram estabelecidos artigos cujo ano de publicação fosse inferior a 2010, além de estudos duplicados nas bases de dados. As pesquisas foram realizadas no período de agosto a setembro de 2021, usando como base de dados: Scientific Eletronic Library Online (SciELO), Google Acadêmico e Literatura Latino-Americana e do Caribe em Ciências da Saúde (LILACS). Como palavras-chaves, foram utilizados os seguintes termos combinados: “cuidado prénatal" e "odontologia".

Inicialmente, foram selecionados 40 artigos, 18 artigos foram excluídos por não se adequar ao critério de inclusão do estudo. Após uma leitura completa dos artigos selecionados, uma amostra de 22 artigos foi utilizada para a elaboração da pesquisa. Ao final foram selecionados 6 artigos para apreciação do exto integral que participaram da revisão sobre o assunto.

A Figura 1 elucida a estratégia de busca e a metodologia de seleção dos artigos.

Figura 1 - Seleção dos artigos utilizados.

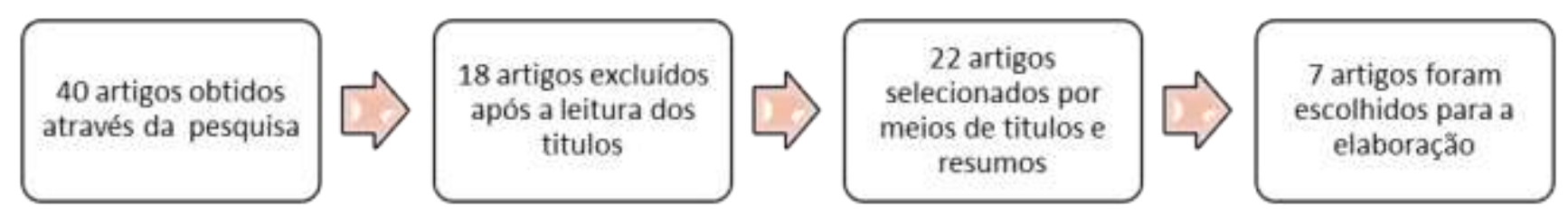

Fonte: Importância do Pré-Natal Odontológico: Uma Revisão Narrativa.

\section{Resultados e Discussão}

A tabela apresenta detalhes dos 7 artigos selecionados. Todos os estudos foram publicados entre 2013 e 2021 , onde foram observados o conhecimento dos Cirurgiões Dentistas sobre a importância do pré-natal odontológico e das gestantes, apesar das gestantes saberem a importância do acompanhamento, algumas ainda relatam receio e dificuldade ao acesso. Mediante as buscas pode-se notar que o tema ainda e pouco abordado. 
Segundo Silva et al. (2020) as gestantes devem ser consideradas um grupo populacional prioritário para atendimento odontológico, pois atuam como agentes multiplicadoras de atitudes e informações dentro do ambiente familiar, influenciando nos hábitos alimentares e de higiene da família, além de constituírem um grupo de fácil acesso, pois passam a frequentar regularmente as unidades de saúde durante esse período, o que as tornam importantes facilitadoras

Tabela 1 - Perfil e característica dos artigos selecionados.

\begin{tabular}{|c|c|c|c|}
\hline ARTIGO & OBJETIVO & $\begin{array}{l}\text { DESENHO DO } \\
\text { ESTUDO }\end{array}$ & CONSIDERAÇÕES FINAIS \\
\hline Lopes et al. (2018) & $\begin{array}{l}\text { Identificar o conhecimento das } \\
\text { gestantes sobre os cuidados } \\
\text { odontológicos na rede pública e } \\
\text { também contribuir para maior } \\
\text { entendimento por parte dos } \\
\text { profissionais de saúde. }\end{array}$ & $\begin{array}{l}\text { Pesquisa de } \\
\text { natureza } \\
\text { qualitativa, do } \\
\text { tipo exploratória. }\end{array}$ & $\begin{array}{l}\text { As gestantes consideram importante o pré-natal } \\
\text { odontológico, sendo uma oportunidade de acesso aos } \\
\text { serviços de saúde bucal. Também associam que a presença } \\
\text { de algumas alterações na boca pode afetar na saúde do bebê. }\end{array}$ \\
\hline Bastiani et al. (2013) & $\begin{array}{l}\text { Avaliar o conhecimento das } \\
\text { gestantes quanto há prevenção, } \\
\text { consequências e oportunidade de } \\
\text { tratamento de possíveis alterações } \\
\text { bucais desenvolvidas na gestação. }\end{array}$ & $\begin{array}{l}\text { Exploratório, } \\
\text { qualitativa e } \\
\text { quantitativa }\end{array}$ & $\begin{array}{l}\text { Foi observado que as gestantes ainda estão bastantes } \\
\text { desinformadas sobre como prevenir as possíveis alterações } \\
\text { bucais que podem ocorrer durante o período gestacional; } \\
\text { também existem crenças e mitos relacionados à gestação e, } \\
\text { embora as gestantes consideram importante o atendimento } \\
\text { odontológico preventivo. Torna-se imprescindível a } \\
\text { instalação educação em saúde para a conscientização das } \\
\text { mães fazendo-se necessário a integração entre a classe } \\
\text { médica e odontológica. }\end{array}$ \\
\hline $\begin{array}{l}\text { Guimarães et } \quad \text { al. } \\
(2021)\end{array}$ & 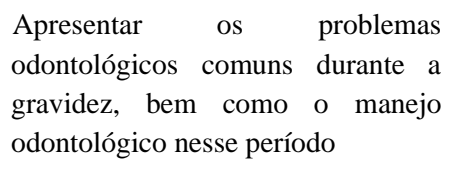 & $\begin{array}{l}\text { Revisão } \\
\text { bibliográfica } \\
\text { qualitativa }\end{array}$ & $\begin{array}{l}\text { O ideal era que fosse realizadas avaliações preventivas antes } \\
\text { da gravidez, o que possibilitaria mais ainda o } \\
\text { desenvolvimento de uma gestação saudável. }\end{array}$ \\
\hline Harb et al. (2020) & $\begin{array}{l}\text { Apresentara importância do pré- } \\
\text { natal odontológico para a população } \\
\text { de um modo geral, já que os } \\
\text { membros da família participam dos } \\
\text { cuidados durante a gravidez e } \\
\text { também serve como fonte de } \\
\text { informação para estudantes de } \\
\text { Odontologia. }\end{array}$ & $\begin{array}{l}\text { Revisão de } \\
\text { bibliográfica } \\
\text { qualitativa }\end{array}$ & $\begin{array}{l}\text { É importante pensar em estratégias para melhorar a } \\
\text { formação do futuro profissional, para um melhor } \\
\text { desenvolvimento de conhecimento dentro das instituições de } \\
\text { ensino. }\end{array}$ \\
\hline Martins et al. (2013) & $\begin{array}{l}\text { Logo, pôde-se constatar que a } \\
\text { assistência odontológica à gestante } \\
\text { é limitada por práticas realizadas } \\
\text { sem aprofundamento e domínio } \\
\text { teórico, fazendo-se necessário } \\
\text { reavaliá-las e instituir protocolos de } \\
\text { atendimento. }\end{array}$ & $\begin{array}{l}\text { Transversal } \\
\text { Quantitativo }\end{array}$ & $\begin{array}{l}\text { Foi observado uma carência a respeito do conhecimento dos } \\
\text { profissionais odontólogos a respeito do pré-natal, } \\
\text { dificultando a conscientização das gestantes sobre tal } \\
\text { importância. E alertando para a importância do } \\
\text { conhecimento teórico e pratico a respeito do assunto. } \\
\text { Enfatiza a importância do gestores em saúde bucal instituir } \\
\text { protocolos de atendimento e repensar as práticas dos } \\
\text { prestadores da assistência odontológica à gestante. }\end{array}$ \\
\hline Botelho et al. (2019) & $\begin{array}{l}\text { Conhecer a percepção das gestantes } \\
\text { sobre a assistência odontológica no } \\
\text { pré-natal. }\end{array}$ & Qualiquantitativo & $\begin{array}{l}\text { As gestantes reconhecem a importância do tratamento } \\
\text { odontológico no período pré-natal, porém, } \\
\text { acompanhamento com o cirurgião-dentista ainda não é uma } \\
\text { rotina nos serviços de saúde investigados. Verificou-se } \\
\text { dificuldade de acesso aos serviços odontológicos }\end{array}$ \\
\hline Silva et al. (2020) & $\begin{array}{l}\text { O objetivo deste artigo é identificar } \\
\text { as produções científicas sobre o } \\
\text { acesso e utilização de serviços } \\
\text { odontológicos por gestantes. }\end{array}$ & $\begin{array}{l}\text { Revisão } \\
\text { Interativa }\end{array}$ & $\begin{array}{l}\text { Apesar do progresso do conhecimento científico-tecnológico } \\
\text { e da evolução da área odontológica, na tentativa de } \\
\text { aprimorar a qualidade de vida do ser humano, a saúde bucal } \\
\text { de gestantes ainda necessita de atenção. }\end{array}$ \\
\hline
\end{tabular}

Fonte: Importância do Pré-Natal Odontológico: Uma Revisão Narrativa. 
Research, Society and Development, v. 10, n. 15, e147101522813, 2021

(CC BY 4.0) | ISSN 2525-3409 | DOI: http://dx.doi.org/10.33448/rsd-v10i15.22813

No estudo de Lopes et al. (2019), de natureza qualitativa, do tipo exploratória, observou-se que a maioria das gestantes entrevistas, um total (90\%) consideram importante a visita ao dentista durante a gestação, sendo que o principal motivo e a prevenção, $(46 \%)$ prevenção e ao tratamento curativo $(21 \%)$ apenas o tratamento curativo (23\%). Observou também que $91,7 \%$ das gestantes acreditam que a alteração na boca durante a gestação poderia influenciar na saúde geral do bebê, porem a minoria não soube falar o que poderia acontecer com a saúde do seu filho. Conclui que mesmo as gestantes tendo aceitação ao tratamento odontológico durante a gestação elas ainda sabem pouco sobre o assunto, falta de conscientização do profissional Médico Agente comunitário de saúde na orientação da gestante sobre o pré-natal odontológico.

Para Bastiani et al., (2013), a maioria das gestantes tinham consciência das necessidades dos cuidados médicos durante a gestação, porém os cirurgiões dentistas fazem parte apenas de $20 \%$ dos programas dos programas do pré-natal. Concordando assim com o Lopes que é necessária mais conscientização das gestantes por parte dos cirurgiões dentistas e faz se necessário ingressar mais cirurgiões dentistas nos programas de saúde com conscientização sobre o atendimento adequado das gestantes no pré-natal assim como uma maior integração entre a classe médica e odontológica visando um melhor esclarecimento sobre a seguridade do tratamento curativo.

Segundo Guimarães et al., (2021) o pré-natal odontológico deve ser iniciado assim que descoberto a gravidez, contudo, o ideal seria a ocorrência de um planejamento prévio à gestação, para que ocorresse a adequação e promoção da saúde bucal, visto que, resultados adversos são observados em gestações que a mãe se encontra em péssimas condições bucais, onde, a prevenção/tratamento de problemas bucais antes do início gestacional seria sinônimo de uma gestação ainda mais saudável, tanto para a mãe como para o filho. O cirurgião-dentista observaria todo o estado de saúde bucal da gestante, o que possibilita o impedimento de agravos de problemas bucais instalados, e a prevenção de novas alterações.

Harb et al., (2020), afirma que ainda existe muita desinformação tanto por parte das gestantes quanto por parte dos profissionais que realizam o seu atendimento, é essencial haver a integração do cirurgião dentista com os outros profissionais que realizam o acompanhamento da gestante.

Martins et al., (2013) conduziu um estudo do tipo transversal quantitativo com os cirurgiões dentistas ativos na rede pública da cidade de Belém no Estado do Para o inquerido foi respondido por 138 profissionais. Através do mesmo foi possível detectar que a maioria dos dentistas já realizaram atendimento odontológico as gestantes, sendo que 12,3 dos profissionais não demostram ter conhecimento sobre o pré-natal. Quanto aos procedimentos realizados, (9\%) dos dentistas afirmaram que realizam os preventivos, sendo eles (palestras, profilaxia e raspagem) e (25\%) realizam procedimento curativos, sendo eles (endodontia, exodontia e cirurgia periodontal) e (52\%) afirmaram que realizam ambos os procedimentos. Assim como Lopes e Bastini, Martins concorda que ainda há uma carência de informações sobre o tratamento exercido pelos profissionais, dificultando a conscientização das pacientes sobre tal tratamento. Afirmando que a assistência odontológica ainda acontece no empirismo.

Botelho et al., (2019), afirma que o tratamento odontológico em gestante ainda e desafiador tanto para as gestantes quanto para os profissionais da saúde. Em sua pesquisa observou-se que $(42,6 \%)$ das gestantes realizam o tratamento odontológico. Assim como os outros autores já citados, Botelho destaca a insegurança no atendimento às gestantes por parte dos profissionais da saúde.

Silva et al. (2020) observou durante seu estudo que um dos motivos pelos quais as futuras mães não realizam o prénatal odontológico se da pelo fato de que muitos cirurgiões dentistas não tem segurança para atendê-las acabam protelando o tratamento odontológico para depois do nascimento do bebê.

O pré-natal odontológico precisa ser expandido, mas para que o mesmo cresça e ajude as futuras mães sobre a importância do mesmo e preciso os profissionais adquiriram conhecimento teórico, prático e cientifico sobre o assunto. 


\section{Conclusão}

O presente estudo abordou vários aspectos e conhecimentos que demonstraram a importância do pré-natal odontológico, e como alguns autores citados argumentam positivamente sobre a importância do acesso aos serviços odontológicos, orientações e aconselhamento precoce sobre a saúde bucal da gestante, enfatizando de que forma a mesma pode influenciar diretamente na saúde geral do bebê.

Além disso, identificou-se que o anestésico local mais indicado para o uso em gestantes com menores riscos de complicações sistêmicas é a lidocaína 2\%, tendo como vasoconstritor adrenalina na concentração de 1:100.000. Se houver a necessidade de aplicação de um anestésico tópico, deve-se dar preferência para o uso da lidocaína tópica $2 \%$.

É extremamente importante estabelecer uma linguagem unificada e de fácil entendimento quando um profissional de saúde precisa orientar a paciente, para que haja êxito na interpretação da informação fornecida, sendo este o caminho que poderá possibilitar novos hábitos que culminarão no bem-estar da mulher e de seu filho. No entanto, apesar de ser um acompanhamento de extrema importância, ainda é pouco executado pelos cirurgiões dentistas, o que aponta para uma maior necessidade na disseminação dessa prática.

\section{Implicações prática}

Sugere-se que realizem mais pesquisa cujo objetivo seja a complementação do presente estudo que apresente infomações alucivas sobre a inserção profissional do Cirurgião Dentista e Equipe multidisciplinar, no âmbito de consultas, orientações e realizações de procedimentos odontologicos durante a gestação com seguraça e embasamento cientifico.

\section{Contribuições dos autores}

MRPS e EDAS concebeu a estratégia de pesquisa em conjunto com PAK. MRPS e EDAS fez as buscas bibliográficas nas bases de dados, a seleção dos artigos e a extração de dados sob a supervisão de PAK. Os autores leram e aprovaram a versão final do manuscrito. O conteúdo da revisão é de exclusiva responsabilidade individuais dos autores.

\section{Declaração de conflito de interesses}

Os autores declaram que a pesquisa foi conduzida na ausência de quaisquer relações comerciais ou financeiras que possam ser interpretados como um potencial conflito de interesse.

\section{Referências}

Alves, T. V., \& Bezerra, M. M. M. (2020). Principais alterações fisiológicas e psicológicas durante o Período Gestacional / Main Physiological and Psychological changes during the management period. Revista De Psicologia, 14(49), 114-126. https://idonline.emnuvens.com.br/id/article/view/2324/3608

Bastiani, C., Cota, A. L. S., Provenzano, M. G. A., Fracasso, M. L. C., Honório, H. H., \& Rio, D. (2013). Conhecimento das Gestantes Sobre Alterações Bucais e Tratamento Odontológico Durante a Gravidez, 12(4), 293-295 http://revodonto.bvsalud.org/pdf/occ/v9n2/a13v9n2.pdf

Botelho, D. L. L., Lima, V. G. A., Barros, M. M. A. F., \& Almeida, J. R. d. S. (2019). Odontologia e gestação: a importância do pré-natal odontológico. SANARE Revista de Políticas Públicas, 18(2). https://sanare.emnuvens.com.br/sanare/article/view/1376

Brasil. (2008). Ministério da saúde. Saúde Bucal. Cadernos de Atenção Básica, 17. Brasília, DF. https://bvsms.saude.gov.br/bvs/publicacoes/saude_bucal.pdf

Carvalho, B., Fritzen, E. L., Parodes, A. G., Santos, R. B., Gedoz, L. (2013). O emprego dos anestésicos locais em Odontologia: Revisão de Literatura. Revista Brasileira De Odontolia. 70(2), 2013. http://revodonto.bvsalud.org/pdf/rbo/v70n2/a16v70n2.pdf

Ebrahim, Z. F., Oliveira, M. C. Q., Peres, M. P. S. M., Franco, J. B. (2014). Tratamento Odontológico em Gestantes. Science In Health, 5(1), 32-44. https://arquivos.cruzeirodosuleducacional.edu.br/principal/new/revista_scienceinhealth/13_jan_abr_2014/Science_05_01_32-44.pdf 
Research, Society and Development, v. 10, n. 15, e147101522813, 2021

(CC BY 4.0) | ISSN 2525-3409 | DOI: http://dx.doi.org/10.33448/rsd-v10i15.22813

Guimarães, K. A., Sousa, G. A., Costa, M. D. M. d. A., Andrade, C. M. d. O., \& Dietrich, L. (2021). Gestação e Saúde Bucal: Importância do pré-natal odontológico. Research, Society and Development, 10(1). https://doi.org/10.33448/rsd-v10i1.12234

Harb, D. A., Carmo, W. D., Boaventura, R. M. (2020). A importância do pré-natal odontológico. Revista Cathedral, 2(3). http://cathedral.ojs.galoa.com.br/index.php/cathedral/article/view/198/62

Kruger, M. S. M., Rosa, D. P., Pappen, F. G., Romano, A. R., Corrêa, F. O. B. (2013). Granuloma gravídico, Odontologia Clínico-Científica (Online), 12(4), 293-295. http://revodonto.bvsalud.org/pdf/occ/v12n4/a11v12n4.pdf

Leal, A. O., Rolim, J. I. A., Muniz, I. A. F., Farias, I. A. P. F. (2013). Estudo dos Parâmetros Salivares de Gestantes. Odontologia Clínico-Científica (Online), 12(1). http://revodonto.bvsalud.org/pdf/occ/v12n1/a09v12n1.pdf

Lopes, I. K. R., Pessoa, D. M. V., \& Macêdo, G. L. de. (2019). Autopercepção do pré-natal odontológico pelas gestantes de uma unidade básica de saúde. Revista Ciência Plural, 4(2), 60-72. https://periodicos.ufrn.br/rcp/article/view/16839/11267

Marla, V., Srii, R., Roy, D. K., \& Ajmera, H. (2018). The Importance of Oral Health during Pregnancy: A Review. MedicalExpress (São Paulo, online), 5. 002 https://doi.org/10.5935/MedicalExpress.2018.mr.002

Martins, L. d. O., Pinheiro, R. D. P. d. S., Arantes, D. C., Nascimento, L. S. d., \& Santos Júnior, P. B. d. (2013). Assistência odontológica à gestante: percepção do cirurgião-dentista. Revista Pan-Amazônica de Saúde, 4(4), 11-18. https://doi.org/10.5123/s2176-62232013000400002

Mascarenhas, V. I., Vilarinho, L. A. L., Moura, L. d. F. A. d. D., Moura, M. S. d., \& Ferro, L. B. (2012). Correlação entre saúde periodontal e idade gestacional. Revista de Odontologia da UNESP, 41(6), 408-414.https://doi.org/10.1590/s1807-25772012000600007

Moimaz, S. A. S., Saliba, O., Santos, K. T., Queiroz, A. P. D. G., Garbim, C. A. S. (2011). Prevalência de Cárie Dentária em Gestantes Atendidas no Sistema único de Saúde em Município Paulista. Revista Odontológica de Araçatuba, 32(1), 44-48. https://apcdaracatuba.com.br/revista/v32n12011/TRABALHO8.pdf

Reis, D. M., Pitta, D. R., Ferreira, H. M. B., Jesus, M. C. P. d., Moraes, M. E. L. d., \& Soares, M. G. (2010). Educação em saúde como estratégia de promoção de saúde bucal em gestantes. Ciência \& Saúde Coletiva, 15(1), 269-276. https://doi.org/10.1590/s1413-81232010000100032

Rodrigues, F., Mármora, B., Carrion, S. J., Rego, A. E. C., Pospich, F. S (2017). Anestesia Local Em Gestantes Na Odontologia Contemporânea. Journal Health NPEPS, 2(1), 254-271. https://periodicos.unemat.br/index.php/jhnpeps/article/view/1835/1675

Silva, C. C. d., Savian, C. M., Prevedello, B. P., Zamberlan, C., Dalpian, D. M., \& Santos, B. Z. d. (2020). Acesso e utilização de serviços odontológicos por gestantes: revisão integrativa de literatura. Ciência \& Saúde Coletiva, 25(3), 827-835. https://doi.org/10.1590/1413-81232020253.01192018

Silva, S. O. (2013). Pré-natal odontológico: importância da educação em saúde para a promoção da saúde bucal no período gestacional, Trabalho de conclusão de curso, Curso de Especialização em Atenção Básica em Saúde da Família, Universidade Federal de Minas Gerais, Belo Horizonte, Brasil.

https://www.nescon.medicina.ufmg.br/biblioteca/imagem/4144.pdf

Silva, S. V. d., Vieira, E. R. L., \& Silveira, P. V. d. (2020). A importância do pré-natal odontológico na prevenção do parto prematuro. Revista Expressão Católica Saúde, 5(1), 77. https://doi.org/10.25191/recs.v5i1.4025

Sousa, J. N. L. d., Nóbrega, D. R. d. M., \& Araki, Â. T. (2014). Perfil e percepção de diabéticos sobre a relação entre diabetes e doença periodontal. Revista de Odontologia da UNESP, 43(4), 265-272. https://doi.org/10.1590/rou.2014.042

Vasconcelos, R. G., Vasconcelos, M. G., Mafra, R. P., Júnior, L. C. A., Queiroz, L. M. G. Barboza, C. A. G. (2012). Atendimento Odontológico a Pacientes Gestantes: Como Proceder com Segurança. Revista Brasileira de Odontologia, 69(1), 120-124. http://revodonto.bvsalud.org/pdf/rbo/v69n1/a27v69n1.pdf 